\title{
A Unique Case of Cutaneous Larva Migrans and Asymptomatic Löeffler's Syndrome
}

\author{
Jason $\mathrm{Ng}^{1}$, Daniel Lee ${ }^{1}$, Marek Kryzstofiak ${ }^{1}$ \\ 1. Internal Medicine, Regional Medical Center Bayonet Point, Hudson, USA \\ Corresponding author: Jason Ng, jasonng18@gmail.com
}

\begin{abstract}
Cutaneous larva migrans is a fairly common parasitic skin disease typically found in more tropical climates such as the southeastern United States, with the most commonly encountered organism known as the hookworm, Ancylostoma braziliense. Löeffler's syndrome is a rare pulmonary manifestation with vague diagnostic criteria but frequently characterized by diffuse migratory pulmonary infiltrates on imaging studies with accompanying serum eosinophilia. Here, we present a unique case of Löeffler's syndrome secondary to cutaneous larva migrans.
\end{abstract}

Review began 06/15/2021 Review ended 06/20/2021 Published 06/27/2021

() Copyright 2021

$\mathrm{Ng}$ et al. This is an open access article distributed under the terms of the Creative Commons Attribution License CC-BY 4.0., which permits unrestricted use, distribution, and reproduction in any medium, provided the original author and source are credited.
Categories: Internal Medicine, Infectious Disease, Pulmonology

Keywords: cutaneous larva migrans, hyper-eosinophilia syndrome, pulmonary disease, infectious and parasitic diseases, parasitic diagnosis, tropical medicine, löeffler’s syndrome

\section{Introduction}

Löeffler's syndrome is a rather rare pulmonary manifestation with vague diagnostic criteria. Upon literature review [1-4], it is frequently characterized as multiple diffuse, migratory pulmonary infiltrates with concomitant serum eosinophilia. Cutaneous larva migrans, on the other hand, is a fairly common tropical parasitic skin disease [2]. In one particular study, $6.7 \%$ of travelers visiting a travel-related clinic presented with this disease [5]. It is typically found in warmer climates where the parasite seeks a host via dermal penetration. Diagnosis is frequently made clinically with careful consideration of both a patient's history and presentation [4]. This case report illustrates the rare pulmonary manifestation in a presentation of cutaneous larva migrans.

\section{Case Presentation}

A 52-year-old Caucasian male presented to our hospital with complaints of ankle pain. He had a serpentine rash over his ankle which had been progressively worsening for the last several weeks with now, diffuse pruritus across his abdomen and chest, which he had been scratching for the aforementioned time period. He denied any insect bites or recent travel outside of the state of Florida but confessed to working outside barefoot in an area where feral cats frequently defecate. The patient did not report any past medical history but did admit to a prior social history including both prior tobacco (30 pack year) and intravenous drug abuse (cocaine and amphetamine). The patient otherwise denied any active recreational drug use or alcohol intake. Upon arrival at the hospital, the patient's vital signs were temperature of $36.9^{\circ} \mathrm{C}$, blood pressure of $121 / 70$ $\mathrm{mm} \mathrm{Hg}$, pulse of 83 beats per minute, and oxygen saturation of $99 \%$ on room air. Multiple areas of serpentine tracks with erythema were noted on physical examination (Figures 1-3). 


\section{Cureus}

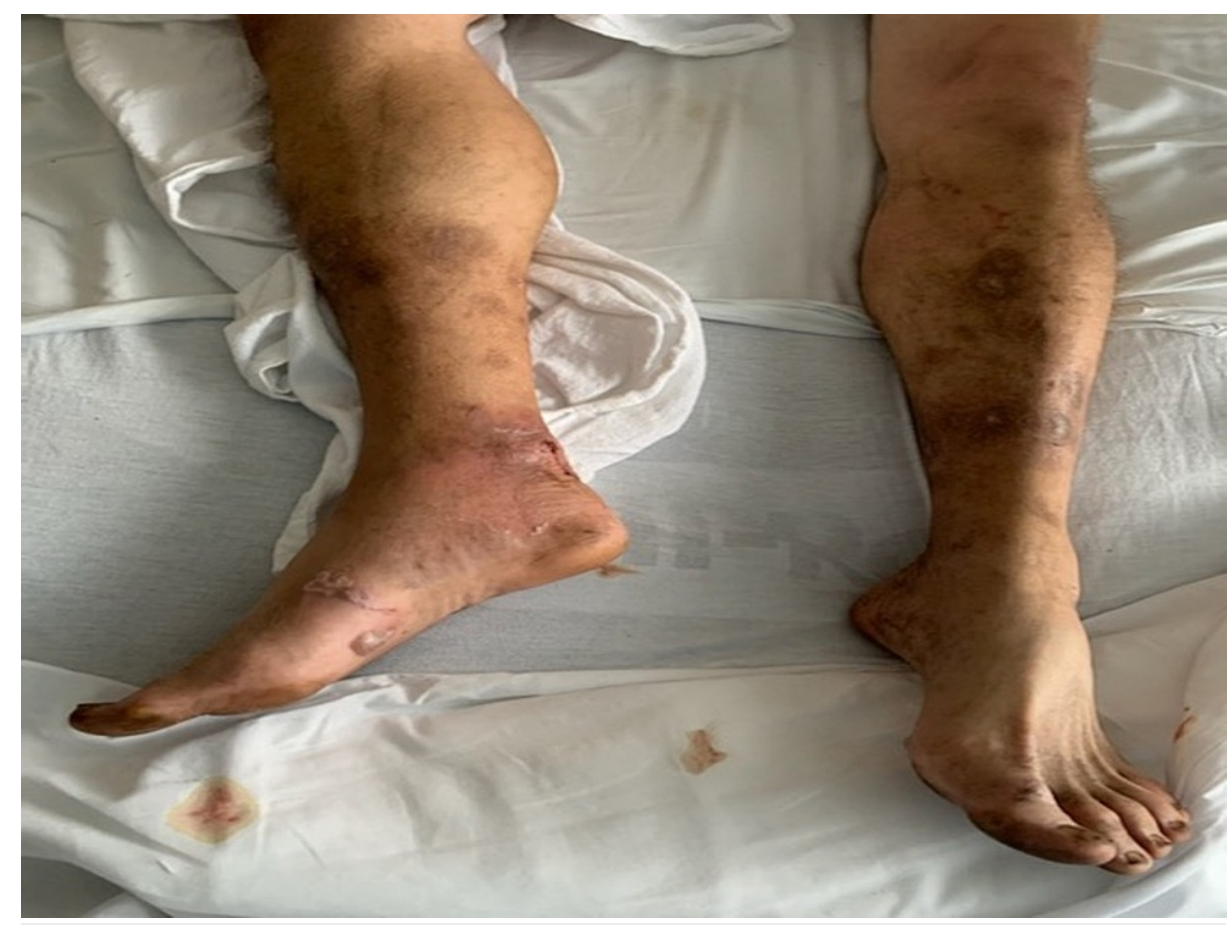

FIGURE 1: Serpiginous rash and bullae over the right foot

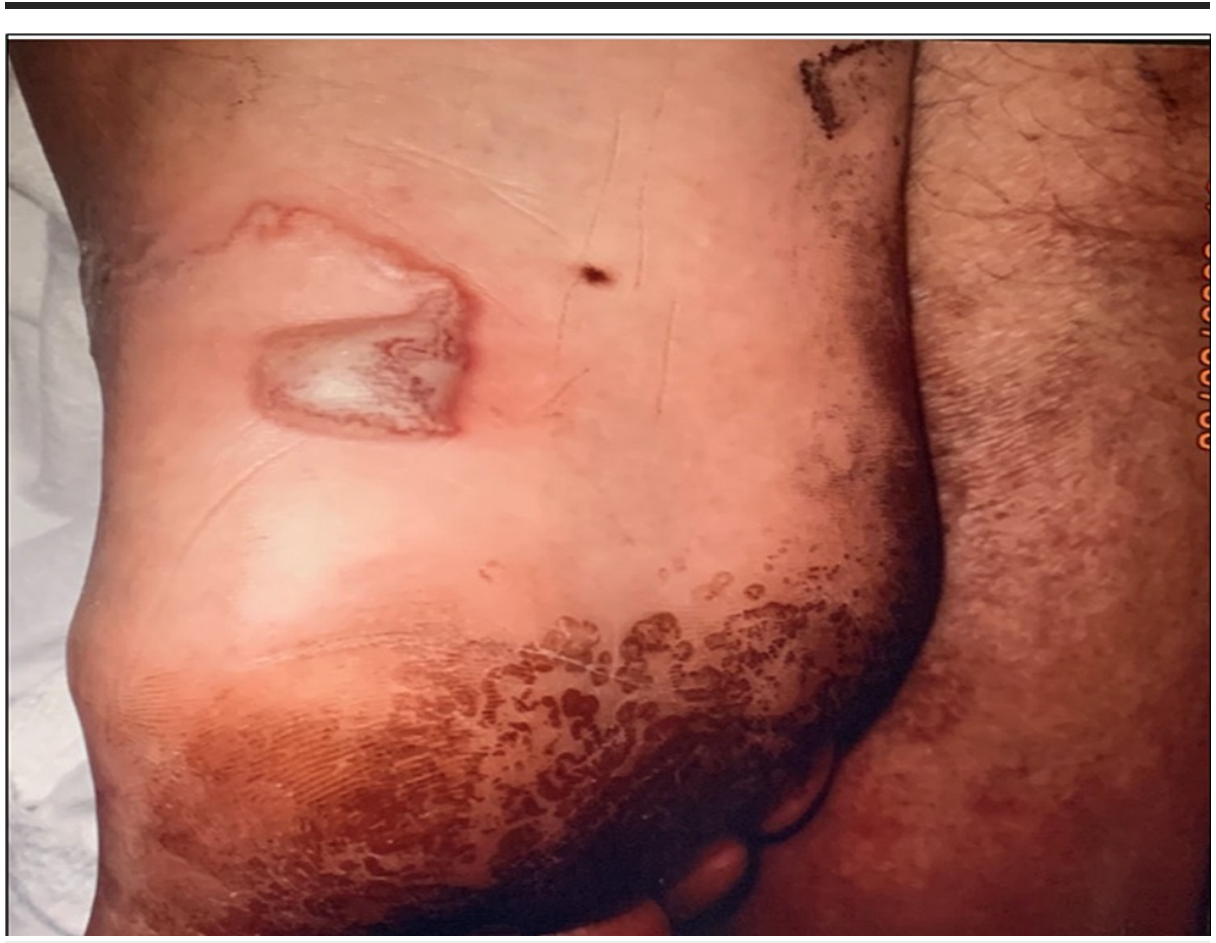

FIGURE 2: Serpiginous lesion with bullae over the right foot 


\section{Cureus}

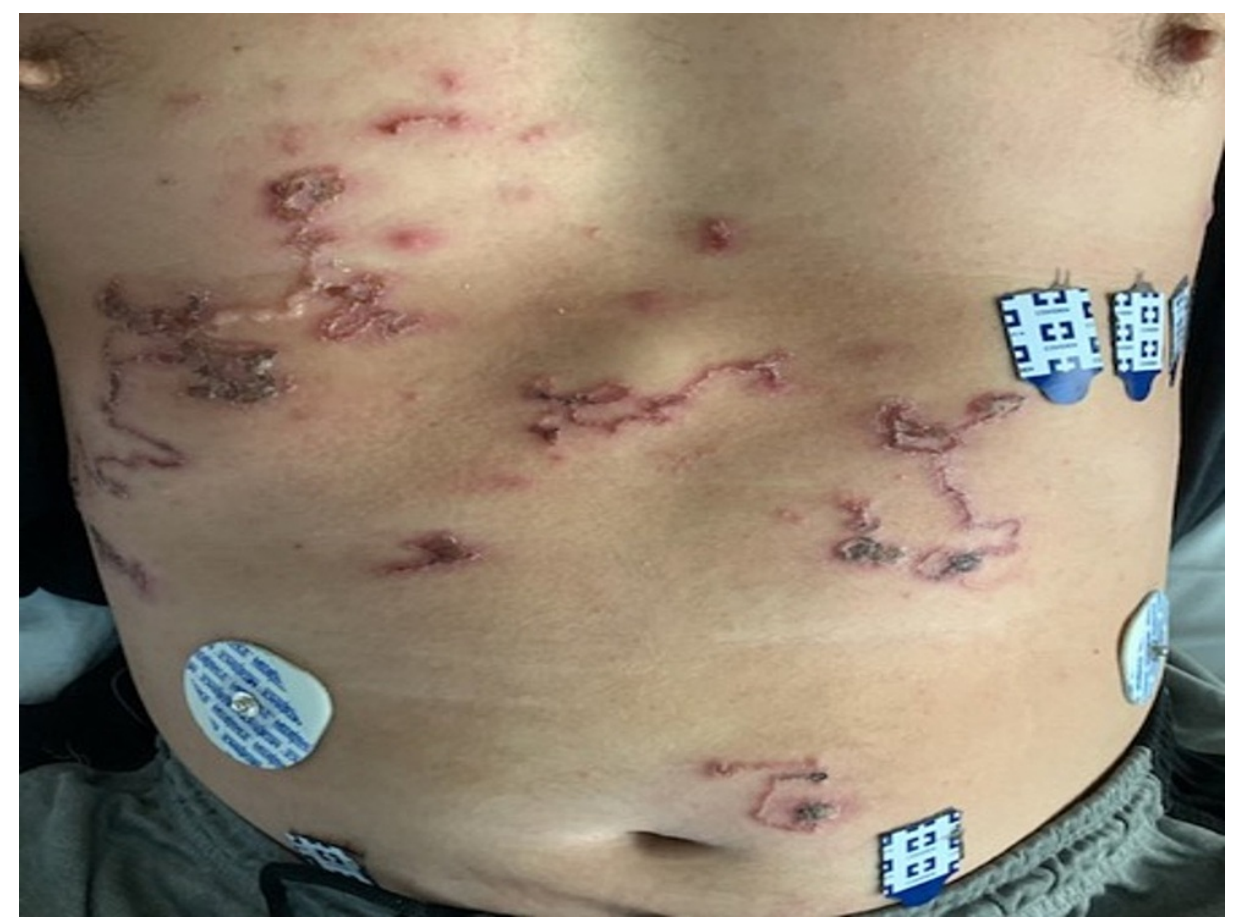

FIGURE 3: Multiple serpiginous lesions with overlying excoriations over the chest and abdomen

Circulating eosinophils in peripheral laboratory studies were elevated at $2.1 \times 10^{3} / \mathrm{uL}(24 \%)$ with a noted immunoglobulin E (IgE) of 1773 (normal < 100). Human immunodeficiency virus (HIV) and stool parasites were negative. Chest radiograph performed in the emergency department incidentally revealed nodular opacities bilaterally (Figure 4). 


\section{Cureus}

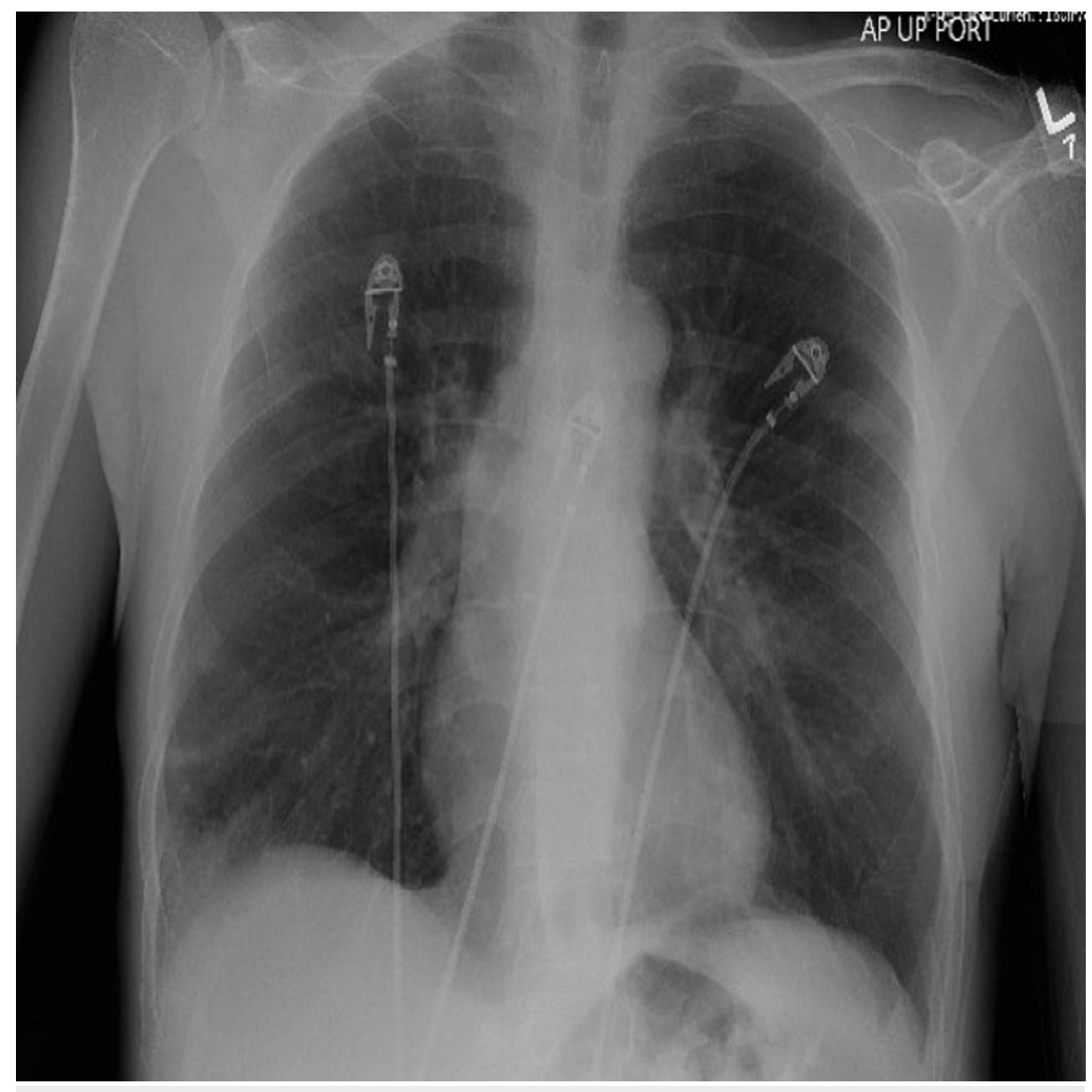

FIGURE 4: Bilateral nodular opacities on chest radiography

A follow-up CT scan showed multiple reticulonodular infiltrates (Figures 5-6). 


\section{Cureus}

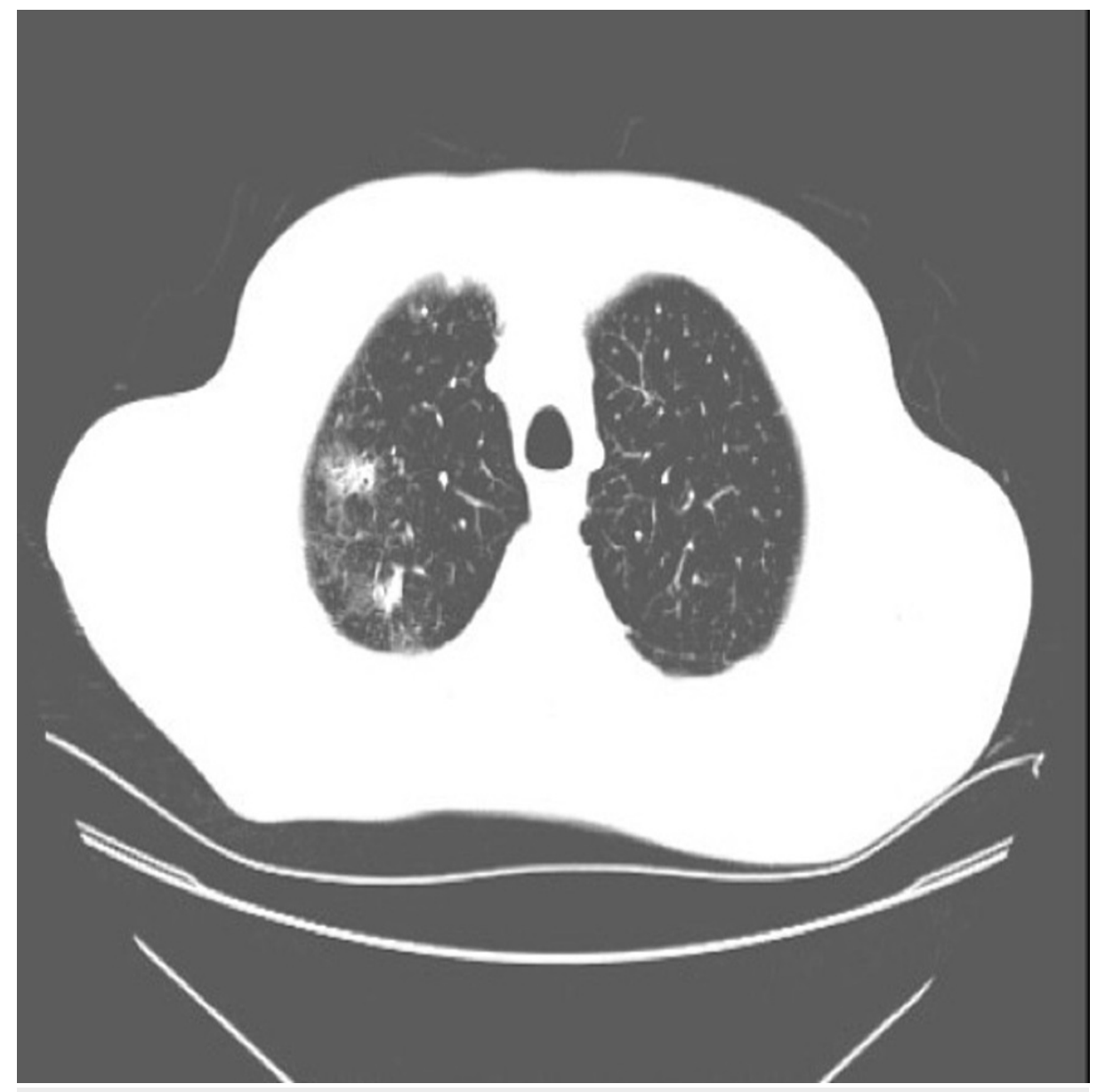

FIGURE 5: Reticulonodular infiltrates in the right upper lobe on CT 


\section{Cureus}

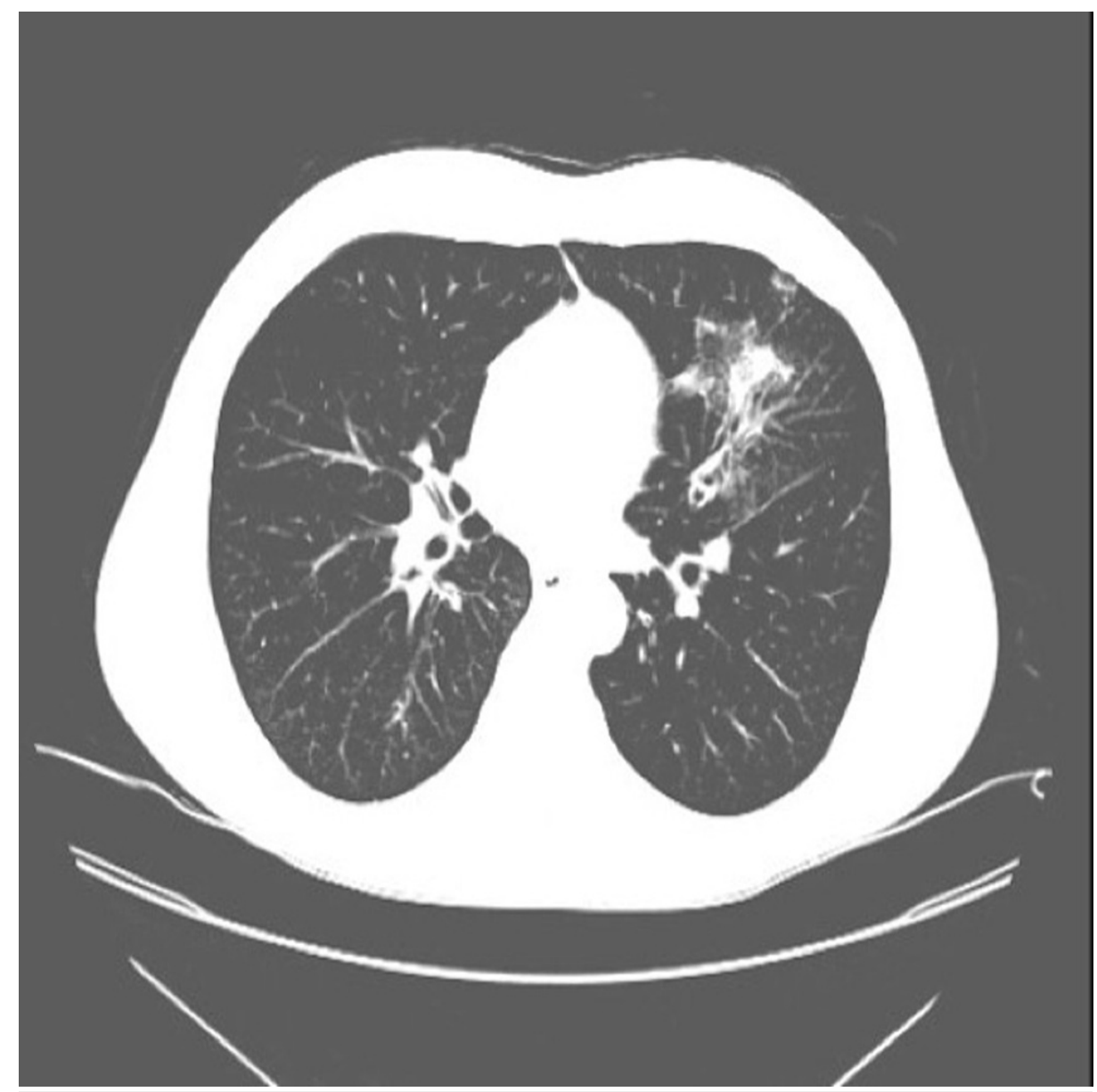

FIGURE 6: Reticulonodular infiltrates on the left lower lobe on CT

The diagnosis of Löeffler's syndrome was made secondary to cutaneous larva migrans given the patient's presentation, laboratory results and findings on radiographic imaging.

The patient was then administered a single dose of oral albendazole $400 \mathrm{mg}$. His dermatological lesions and severe pruritus improved. The patient fortunately continued to not have any respiratory complaints with oxygen saturations remaining stable on room air. He was ultimately discharged home with a course of oral doxycycline due to concerns of superimposed cellulitis with instructions to followup with an outpatient facility for routine surveillance of his serum eosinophilia and pulmonary infiltrates.

\section{Discussion}

Cutaneous larva migrans is a condition secondary to the migration of parasites with one of the most common being Ancylostoma braziliense [4] in the southeastern United States. The hookworm lives in the gastrointestinal systems of both felines and canines with the parasitic eggs frequently found in their feces. These organisms then go on to infect their host via dermal invasion. Barefoot individuals seem to be most at risk, as they account for up to $95 \%$ of cases [3]. Shortly after inoculation, serpentine tracks soon develop as the nematode burrows which then allows it to travel anywhere from a couple of millimeters to several centimeters per day [2,3]. Fortunately, the organism cannot complete its full life cycle in humans and usually dies within months of inoculation.

The diagnosis is made clinically after a careful review of the patient's history. Given the very distinct clinical features, other dermatological conditions can effectively be ruled out. Biopsy is rarely useful since the parasite itself is often located beyond the superficial lesions making it very difficult to isolate [4,6]. Stool samples are also not helpful until there is gastrointestinal involvement [1].

The disease itself is self-limiting but anti-helminthics are commonly administered due to the severe accompanying pruritus. Pharmacologic options include oral albendazole $400 \mathrm{mg}$ or ivermectin $200 \mathrm{mcg} / \mathrm{kg}$. Oral albendazole is well tolerated due to its overall low dosage and relatively fast anti-helminthic activity. Regression of lesions is typically seen in 24 to 48 hours [7]. Oral ivermectin is another well-tolerated therapy but upon literature review, it appears to be slightly less efficacious for specific cases of cutaneous larva 
migrans with associated folliculitis [6]. The duration of both therapies can be adjusted depending on the severity of the patient's symptoms. Other options are available but found to be not nearly as effective as the aforementioned ones. Success with cryotherapy is rather limited due to both the difficulty in localizing the organism and its inherent ability to survive low temperatures. Topical anti-helminthics are another option but local irritation can occur due to the need for repeat applications. Recurrence rates also tend to be higher, especially in cases with widespread lesions as opposed to localized ones [7]. Finally, antibiotics are typically warranted since overlying bacterial folliculitis are fairly commonplace in these infestations [3].

Löeffler's syndrome is a type of pulmonary eosinophilia syndrome commonly caused by parasites. In this specific instance, it is thought that the pulmonary manifestations arise secondary to a type one hypersensitivity reaction as the larvae migrate to the lung parenchyma, leading to massive activation of eosinophils $[1,3]$. In its asymptomatic form, it is usually self-resolving requiring no treatment. Symptomatic patients may be given extended courses of both anti-helminthics and corticosteroids. Serial chest imaging and laboratory studies should be obtained to ensure resolution, although this can take anywhere from weeks to several months.

\section{Conclusions}

In summary, Löeffler's syndrome should be considered in the differential diagnosis where patients present with migratory pulmonary infiltrates, peripheral eosinophilia, and skin findings. Diagnosis is typically made clinically due to the overall vague diagnostic criteria, so the patient's history and presenting symptoms are of utmost importance. Treatment is typically not needed but can be tailored to the individual depending on their presentation, and severity of both their dermatologic and pulmonary manifestations.

\section{Additional Information \\ Disclosures}

Human subjects: Consent was obtained or waived by all participants in this study. Conflicts of interest: In compliance with the ICMJE uniform disclosure form, all authors declare the following: Payment/services info: All authors have declared that no financial support was received from any organization for the submitted work. Financial relationships: All authors have declared that they have no financial relationships at present or within the previous three years with any organizations that might have an interest in the submitted work. Other relationships: All authors have declared that there are no other relationships or activities that could appear to have influenced the submitted work.

\section{References}

1. Ekin S, Sertogullarindan B, Gunbatar H, Arisoy A, Yildiz H: Loeffler's syndrome: an interesting case report . Clin Respir J. 2016, 10:112-4. 10.1111/crj.12173

2. Gao YL, Liu ZH: Cutaneous larva migrans with Löeffler's syndrome. Am J Trop Med Hyg. 2019, 100:487-488. 10.4269/ajtmh.18-0406

3. Podder I, Chandra S, Gharami RC: Loeffler's syndrome following cutaneous larva migrans: an uncommon sequel. Indian J Dermatol. 2016, 61:190-2. 10.4103/0019-5154.177753

4. Te Booij M, de Jong E, Bovenschen HJ: Löffler syndrome caused by extensive cutaneous larva migrans: a case report and review of the literature. Dermatol Online J. 2010, 16:2. 10.5070/D34g15v8vm

5. Ghosh SK, Bandyopadhyay D: Dermacase: can you identify this condition? Cutaneous larva migrans . Can Fam Physician. 2009, 55:489-491.

6. Vanhaecke C, Perignon A, Monsel G, Regnier S, Bricaire F, Caumes E: The efficacy of single dose ivermectin in the treatment of hookworm related cutaneous larva migrans varies depending on the clinical presentation. J Eur Acad Dermatol Venereol. 2014, 28:655-7. 10.1111/jdv.12097

7. Albanese G, Venturi C, Galbiati G: Treatment of larva migrans cutanea (creeping eruption): a comparison between albendazole and traditional therapy. Int J Dermatol. 2001, 40:67-71. 10.1046/j.13654362.2001.01103.x 